\title{
Artículo Original \\ Efecto de la torta de Sacha inchi (plukenetia volúbilis l.) en el perfil bioquímico sanguíneo e histopatología del hígado de aves de postura
}

\author{
[Sacha inchi (plukenetia volúbilis I.) cake effect on blood biochemical profile \\ and liver histopathology of laying hens]
}

\author{
Leidy L. Hurtado-Ramírez ${ }^{1}$, Daniel Paredes-López² ${ }^{2}$ Rizal Robles-Huaynate ${ }^{2 *}$ \\ 1'Área de Asistencia Técnica, Municipalidad del distrito de Pomahuasi, Leoncio Prado, Huánuco. \\ 2Área de Sanidad Animal, Departamento de Ciencia Animal, Universidad Nacional Agraria de la Selva, Av. Universitaria \\ s/n, PO Box 156, Tingo María. \\ *e-mail: rizalcides@gmail.com
}

\begin{abstract}
Resumen
La torta de sacha inchi es un insumo de alto valor proteico para la alimentación animal, sin embargo, contiene elevado contenido de factores antinutricionales lo cual limita su uso. El objetivo de esta investigación fue determinar los efectos de la torta de sacha inchi (TSI) en el perfil bioquímico sanguíneo e histología del hígado de gallinas de postura en la etapa de levante. Para esto se utilizó 64 pollitas de 30 días de edad, de la línea Hy Line Brown las que fueron divididas en 4 tratamientos T0, T1, T2 y T3 y alimentados con $0 \%, 6 \%, 10 \%$ y $14 \%$ de TSI respectivamente. Las muestras de sangre fueron obtenidas a los 30,44 , 72 y 100 días de edad; el diseño estadístico usado fue el DCA con arreglo factorial $4 \times 3+1$. Los niveles de TSI en la dieta mostró efecto sobre los niveles del hematocrito, hemoglobina y $\operatorname{ALT}(p<0,05)$. En el tejido hepático en el T3 se observó mayor concentración de hepatocitos con disminución del lumen de los espacios sinusoides e incremento de núcleo en los hepatocitos a comparación del T1. En conclusión, la TSI cambió los niveles de hematocrito, hemoglobina y ALT y tuvo efecto hepatotóxico en las gallinas.
\end{abstract}

Palabras claves: Sacha inchi, aves de postura, ración, perfil bioquímico, hígado.

\begin{abstract}
The objective of this research was to determine the effect of sacha inchi cake (SIC) on the serum biochemical profile, liver relative weight and histology of laying hens. For this purpose 64, 2-day old pullets were divided in 4 treatments T0, T1, T2 and T3, fed $0 \%, 6 \%, 10 \%$ and $14 \%$ of SIC respectively. Blood was sampled at 30, 44, 72 and 100 days old; a complete randomized design (CRD) with 4x4+1 factorial arrangement was used. Levels of SIC shown effect $(p<0.05)$ on hemoglobin, hematocrit and ALT, but these results were between normal levels. Liver tissue showed polynuncleated hepatocytes in T2 and T3. Conclusively, SIC changed the hematocrit, hemoglobin and ALT levels and had a negative effect in liver tissue in laying hens.
\end{abstract}

Key words: Sacha inchi, Plukenetia volúbilis L, biochemical profile, laying hens. 


\section{INTRODUCCIÓN}

Actualmente la avicultura se encuentra centralizada en la costa y en el norte del país, debido a esto, el abastecimiento de carne y huevos en nuestra región es proveída de estas dos regiones. Sin embargo, nuestra región cuenta con excelentes condiciones climatológicas y ambientales, con insumos alimenticios regionales que podrían ser utilizados en la alimentación de aves como la torta de sacha inchi obtenida como residuo después de la extracción de aceite de la semilla, caracterizada por su alto valor proteico (59.13\%) (Brioso, 2007). Así la torta de sacha inchi surge como una alternativa para sustituir a insumos proteicos, los cuales conllevan a la disminución de los costos de producción y así ofertar productos a mejores precios.

Con fines de dar uso al producto residual se ha incluido en la ración de algunos monogástricos, por el alto contenido proteico y disponibilidad de la torta de sacha inchi, suministrándose porcentajes mínimos en la ración según el requerimiento enérgicoproteico del animal (Quispe, 2008; Quintana, 2009; Torres, 2010). Sin embargo, las semillas de sacha inchi contienen factores antinutricionales (Mondragón, 2009; Castillo, et al. 2010) que intervienen en la digestibilidad y metabolicidad. Al incrementar los niveles de sacha inchi en la dieta, se incrementaría los niveles de factores antinutricionales (FAN) lo que podría causar cambios en el peso y estructura histológica del hígado, variación de los niveles de proteína total, albumina, hemoglobina, hematocrito y transaminasas: Aspartato aminotransferasa (AST) y Alanina aminotransferasa (ALT) en sangre de aves de postura en la etapa de levante. El objetivo del presente trabajo fue determinar el efecto de diferentes niveles de torta de sacha inchi sobre los perfiles bioquímicos sanguíneos, el peso y estructura histológica del hígado en gallinas de postura en la etapa de levante.

\section{MATERIALES Y MÉTODOS}

\section{Área de investigación y animales}

El presente trabajo de investigación se ejecutó en la instalación de la unidad experimental de aves y en el Laboratorio de Sanidad Animal de la Facultad de Zootecnia de la Universidad
Nacional Agraria de la Selva, en Tingo María. Se usó 64 pollitas de 30 días de edad, de la línea Hy Line Brown. Las aves fueron criadas bajo las mismas condiciones de alimentación, manejo y control sanitario.

\section{Preparación de las raciones}

Las raciones se formularon tomando como referencia los requerimientos nutricionales para aves de postura propuestos por Hy Line Brown. (2011). El valor nutricional de las raciones usadas fueron de $19.16 \%$ de proteína total para aves de 4 a 12 semanas de edad y $18.38 \%$ para la fase entre 13 a 16 semanas; así mismo el contenido de energía fue de $3045.9 \mathrm{kcal} / \mathrm{Kg}$ para la fase $4-12$ y de $2900.9 \mathrm{Kcal} / \mathrm{Kg}$ para la fase de $13-16$ semanas de edad.

\section{Recolección y procesamiento de muestras}

Los animales fueron muestreados a los 30, 44, 72 y 100 días de vida a tempranas horas y en ayunas, la extracción de sangre fue de la vena alar. El hematocrito se determinó mediante microhematocrito en una centrífuga Hettich EBA 20, y la hemoglobina se determinó por el método de cianometahemoglobina; la alanina aminotransferas (ALT), aspartato aminotransferasa (AST), albúmina y proteína sérica se determinó mediante reactivos de Wiener Lab y para las lecturas se usó un espectrofotómetro Boeco Germany S-22 UV/visible.

El peso del hígado se realizó a los 100 días de edad una vez sacrificadas las aves, se usó una balanza electrónica con precisión decimal $(0,1 \mathrm{~g})$. Se usó 4 pollitas por tratamiento; los cuales eran los que tenían los pesos intermedios dentro de un mismo corral. Para la evaluación histológica se extrajo muestras de hígado y luego se fijaron en solución de formol al 10\% (Alzola, 2001).

Para el análisis de los resultados se usó el diseño completamente al azar (DCA) con arreglo factorial $4 \times 4+1$ (Cuatro niveles de TSIP y 4 tiempos +1 tiempo); los datos fueron analizados mediante el análisis de variancia, para el caso del perfil bioquímico sanguíneo. Para evaluar el de peso relativo del hígado se utilizó el DCA simple. Para el cálculo de las diferencias significativas 
mínimas entre las medias del tratamiento se utilizó el test de Student-Newman-Keuls (SNK).

\section{RESULTADOS Y DISCUSIONES \\ Efecto del consumo de la torta de Sacha Inchi precocida sobre el perfil bioquímico sanguíneo de aves de postura en la etapa de levante.}

En el perfil bioquímico sanguíneo de aves de postura (Cuadro 1), bajo el efecto de la TSI no mostraron efecto sobre los perfiles de albúmina, proteína sérica y AST de los pollos, mientras que en los niveles de hematocrito, hemoglobina y ALT si tuvo efecto $(p<0,05)$. En cuanto al efecto de la edad, los perfiles evaluados resultaron ser estadísticamente diferentes $(p<0,05)$.

Los niveles de hemoglobina a diferentes niveles de TSI, son estadísticamente diferentes $(p<0,05), 8.96 \mathrm{~g} / \mathrm{dl}$ para el T0 y $9,94,10,00$ para el T1 y T2 respectivamente. Este aumento inicial en las concentraciones de hemoglobina para los tratamientos que tienen 6 y $10 \%$ de TSI en la dieta se debe probablemente a la proliferación de eritrocitos los que contienen a esta proteína básicamente para la oxigenación. Así mismo si se hubiese observado una disminución de esta proteína podría deberse a una hemolisis ya que la saponina presente en la TSI tiene acción hemolítica en la sangre, pero este efecto se reduce al ser suministrado por vía oral, debido al bajo nivel de absorción por el tubo digestivo dependiente de la edad del animal (Gómez, 1997).

Los niveles de hematocrito de gallinas obtenidos bajo los efectos de los diferentes niveles de TSI, resultantes de las evaluaciones muestran diferencia estadística $(p<0,05)$ $27,25 \%$ para el $\mathrm{T0}, 30,13 \%$ y $30,47 \%$ para los tratamientos 1 y 2 respectivamente, observándose un aumento según el nivel de inclusión de TSI; esto puede deberse a que el organismo de las aves desarrolla una oxigenación compensatoria frente al efecto marcado de los FAN contenidos en la TSI, desarrollando así una hiperplasia eritroide en la sangre. El nivel normal de hematocrito en aves es de 23 a 55\% (Universidad de Zaragoza, 2011).

Con respecto a la edad del ave, los niveles de hematocrito resultaron ser estadísticamente diferentes ( $p<0.05)$; como se observa el hematocrito aumenta de acuerdo a la edad siendo 26,$63 ; 30,47 ; 29,78$ y $29,88 \%$ a los 30, 44, 72 y 100 días de edad respectivamente; siendo los más altos y estadísticamente iguales entre sí a los 44 y 72 días de edad esto concuerda con lo reportado por Mateo (2006), los valores de hematocrito dependen de la edad y del sexo, siendo más elevados en edades adultas y/o en machos.

Los niveles de proteína sérica y albúmina, resultaron ser estadísticamente iguales ( $p>$ $0,05)$ y encontrándose dentro de los parámetros normales en ambos casos. En el presente estudio en el que se reporta una hiperplasia del hígado, éstos resultados concuerdan con las concentraciones de proteína y albúmina que por lo general son normales en enfermedades hepáticas crónicas (Ferato, 2010). Así mismo los resultados obtenidos para proteína sérica y albúmina concuerdan con lo hallado por Perozo, et al., (2003) quien reportó 3,18 $\pm 0,42$ de proteína total. Por su parte Miranda et al. (2007), alimentando pollos con harina de granos de fríjol (Vigna unguiculata L.) reportó para el tratamiento control $1,66,1,92$ y $1,61 \mathrm{mg} / \mathrm{dL}$ a la 1ra, 2da y 3ra semana de vida, no encontrando diferencia estadística ( $p<0,05$ ). Con respecto a la edad del ave, los niveles de albúmina y proteína obtenidas en diferentes periodos de vida de las aves en estudio son estadísticamente diferentes $(P<0.05)$; observándose un mayor valor a los 72 días comparado a los 30 días de vida.

Los niveles de ALT son estadísticamente diferentes $(p<0,05)$; en el T0 ( $0 \% \mathrm{TSI})$, se observa que la concentración de ALT es de 29.8 siendo mayor que los tratamientos 1,2 y 3 que muestran niveles de 25,$2 ; 22,6$ y 27,1 UI/L respectivamente. Esta disminución en la concentración de la ALT, podría deberse también al incremento de desarrollo de tejido hepático, causado por los diferentes factores antinutricionales que posee la TSI los cuales afecta a los hepatocitos generando una sobrepoblación de hepatocitos muy jóvenes. Así mismo los datos de ALT obtenidos están dentro de los niveles normales 9,5 a 37,2 UI/L (Miranda et al., 2007). Por otra parte Jínez et al. (1998) realizando un estudio sobre el efecto de niveles elevados $(10 \%, 20 \%$ y $30 \%$ ) de semilla de Jamaica (Hibicus 
sabdariffa) en dietas para pollos sobre el comportamiento productivo y funcionamiento hepático; concluyó que no hubo ningún efecto de la semilla de Jamaica sobre la concentración de ALT, basándose en que lo datos registrados cayeron dentro de los niveles normales (70 -220 UI/L).

Los niveles de aspartato aminotransferasa AST (Cuadro 1) bajo el efecto de los niveles de TSI de las aves en estudio son estadísticamente iguales ( $p<0,05)$; esto probablemente debido a que esta enzima es secretada por diferentes tejidos y no particularmente por el tejido hepático; por lo que a pesar del incremento de la mitosis de los hepatocitos (hiperplasia) ocasionado por glucósidos u otros FAN de la TSI, no existe una variación significativa en sangre. Los valores normales de de AST en pollos es de 70 a 220 UI/L (Jínez et al., 1998).

Reátegui (2012) alimentando pollos de carne con 7 y $14 \%$ de TSI hasta los 47 días de edad, no encontró alteraciones en el perfil bioquímico sanguíneo probablemente debido al corto periodo del ciclo de producción del pollo pero en cuanto al hígado, si encontró hiperplasia y esto puede deberse a que este órgano posee mayor capacidad de respuesta frente a la agresión tóxica, debido a su función clave como vía primaria de desintoxicación. En el presente trabajo es probable que el organismo de las aves de los tratamientos 7 y $14 \%$ de TSI, emplearon los pocos nutrientes absorbidos para mantener su bioquímica y fisiología en un correcto nivel; los nutrientes obtenidos del alimento tiene como función primaria satisfacer sus actividades vitales tales como el mantenimiento de la homeostasis corporal, la regeneración celular, etc. que son vitales y prioritarias para la vida, pero no suponen ninguna producción. La mencionada función fue eficiente por la corta exposición a las saponinas y glucósidos de la TSI.

\section{Efecto del consumo de diferentes niveles de TSIP en la ración sobre el tejido hepático.}

El peso relativo del hígado se encontró diferencia estadística significativa entre los atamientos T0, T1, T2 y T3 que mostraron los resultados 1,$55 ; 2,53 ; 3,24$ y $2,56 \%$ respectivamente, interpretándose que a mayor concentración de TSI en la dieta, el peso relativo del hígado tiende a aumentar; esto probablemente producto de una hipertrofia por la exigencia del hígado en producir transaminasas para el metabolismo aminoacídico de la proteína presente en la ración. El cuadro 2 y figura 1 muestran la descripción de la histología hepática de gallinas de postura a los 100 días de edad alimentados con torta de sacha inchi, donde se observa que el T3 presenta una mayor concentración de hepatocitos esto probablemente se debe a que una de las causas más comunes de hiperplasia es la irritación crónica por presencia de toxinas lo cual provoca la proliferación y el acumulo de las células; una de las toxinas que pudo causar la hiperplasia hepática son los glucósidos (Mondragón, 2009), la presencia de glucósidos en la torta de sacha inchi es abundante $(+++)$; los glucosinolatos al ser hidrolizados generan glucosa, ácido sulfúrico y compuestos volátiles como isotiocianatos, oxazolidin-2-tionas, tiocianatos y nitrilos, causando estos últimos lesiones en el hígado.

Bafna et al., (2005) citado por Castillo et al., (2010) menciona que el hígado posee mayor capacidad de respuesta frente a la agresión toxica, debido a su función desintoxicante; esto mismo puede ser causa de su propia lesión, ya que en la biotransformación de sustancias tóxicas pueden generarse metabolitos secundarios, los que en ocasiones pueden producir lesiones hepatocelulares. Los efectos de la intoxicación por el contenido de glucósidos en las aves de corral son varias; siendo una de ellas la lesión hepática (Cornell University, 2009), pudiendo haber sido el resultado de una intoxicación por glucósidos contenidos en la ración dada.

Por lo que la TSI posee factores antinutricionales o sustancias nocivas, ya que la estructura histológica hepática en los pollos del $\mathrm{T} 1, \mathrm{~T} 2$ y $\mathrm{T} 3$ resulto afectada a comparación del T0. Cabe resaltar, que los parámetros bioquímicos sanguíneos en aves de postura de la línea Hy-line variedad, siendo esta investigación una de las primeras. 
Cuadro 1. Efecto del consumo de TSI y edad sobre el perfil bioquímico sanguíneo de aves de postura HyLine Brown en la etapa de levante.

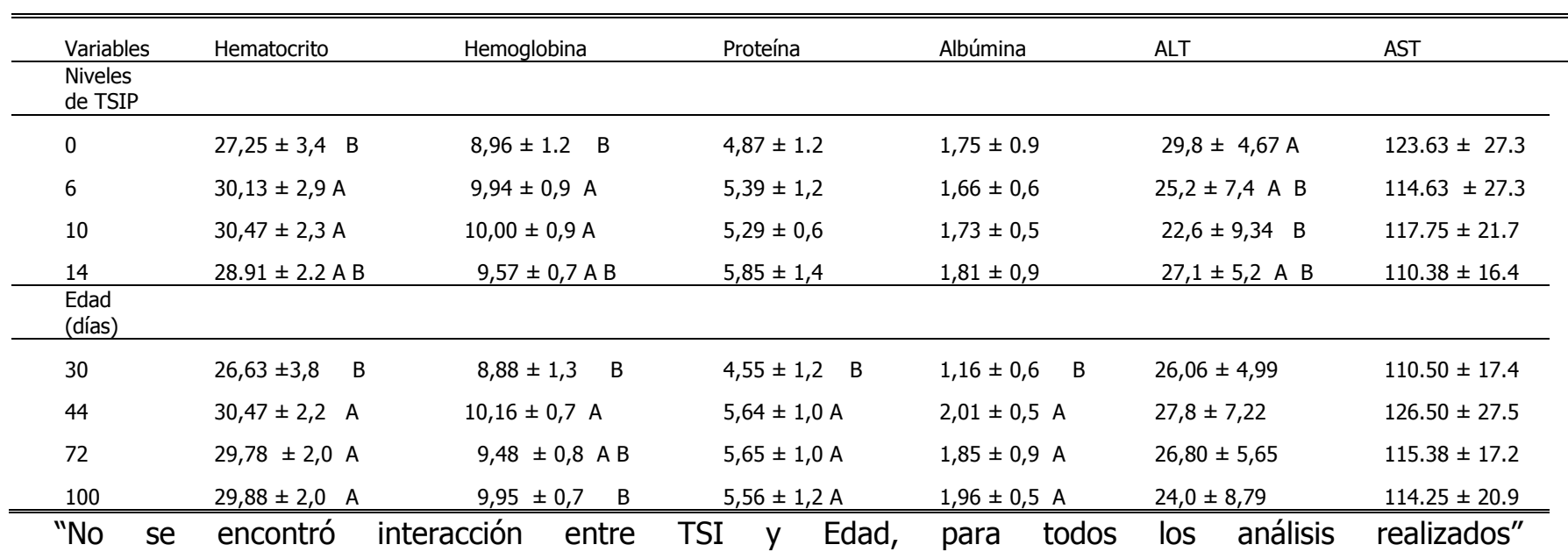

Cuadro 2. Estructura histológica del tejido hepático de pollos a los 48 días de edad alimentados con torta de sacha inchi.

\begin{tabular}{|c|c|c|c|}
\hline Partes & T1 (0\% TSIP) & T2 (7\% TSIP) & T3 (14\% TSIP) \\
\hline $\begin{array}{l}\text { Sinusoides } \\
\text { spáticos }\end{array}$ & Arquitectura normal & $\begin{array}{l}\text { Efectos no muy } \\
\text { arcados }\end{array}$ & Luz sinusoidal disminuida \\
\hline Hepatocitos & $\begin{array}{c}\text { Arquitectura normal } \\
\text { heville, 1994) }\end{array}$ & $\begin{array}{l}\text { Aparición } \\
\text { spatocitos } \\
\text { slinucleados }\end{array}$ & $\begin{array}{l}\text { Aumento considerable de volumen de } \\
\text { s hepatocitos polinucleados }\end{array}$ \\
\hline
\end{tabular}
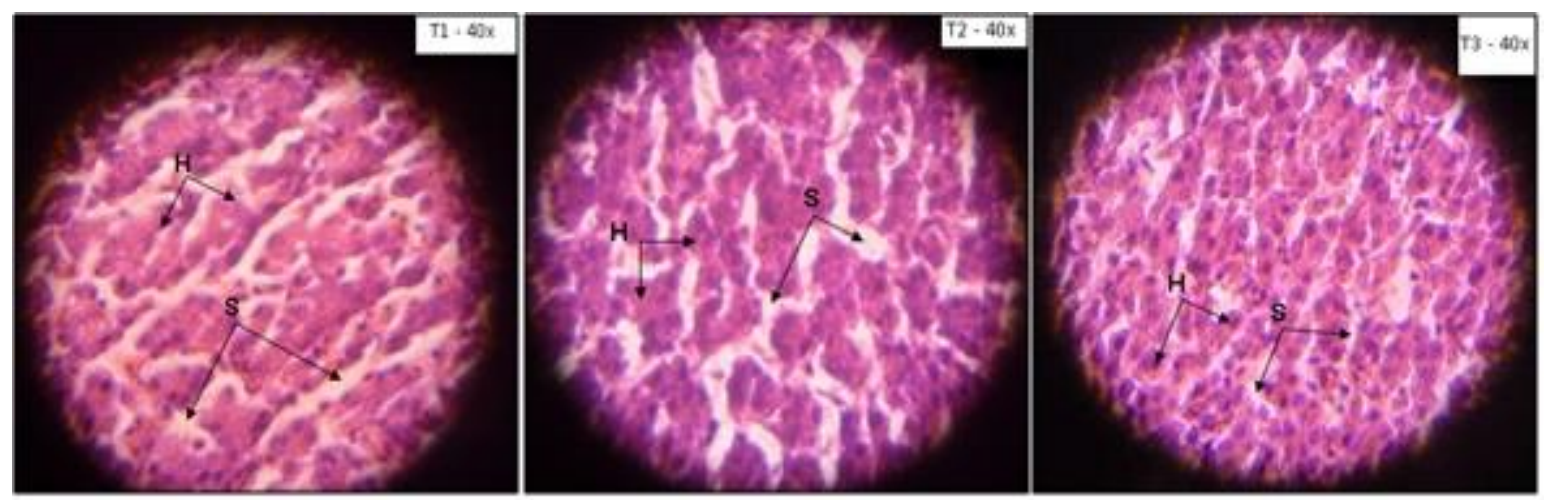

Figura 1. Fotografías de la estructura histológica del tejido hepático según la inclusión de TSI. H: Hepatocito, S: Sinusoide hepático

\section{CONCLUSIÓN}

Los niveles de TSI incluidos en la ración de aves de postura en la etapa de levante produjeron variación en el perfil bioquímico sanguíneo para hemoglobina, hematocrito y
ALT. El peso relativo del hígado aumentó conforme al nivel de inclusión de TSI en la ración; a nivel celular disminuyó el lumen de los espacios sinusoidales del hígado e incremento de núcleos en los hepatocitos. 


\section{AGRADECIMIENTOS}

Al Sr. Félix Jara Ramírez, Laboratorista del laboratorio de Sanidad Animal de la Facultad de Zootecnia, por su apoyo en los análisis realizados. $Y$ al Ing. Hugo Saavedra Rodríguez, por su apoyo en el manejo de las aves.

\section{REFERENCIA BIBLIOGRAFICA}

Alzola R. 2001. Curso de Histología, Embriología y Teratología. Técnicas Histológicas. UNCPBA (Facultad de Ciencias Veterinarias). [En línea]: Vet.unicen.edu, http://www.vet.unicen.edu.ar/html/Areas /Documentos/ Tecnicashistologicas.pdf [Consultado 01 diciembre 2011].

Brioso B. 2007. Evaluación del valor nutricional y energía metabolizable del sacha inchi (Plukenetia volubilis L Walp) integral en pollos de carne. Tesis de Ing. Zootecnista. Universidad Nacional Agraria de la Selva, Tingo María. 45pp Castillo, E; Castillo, S; Reyes, E. 2010. Estudio fitoquímico de Plukenetia volubilis L. y su efecto antioxidante en la lipoperoxidación inducida por Fe3+ / ascorbato en hígado de Rattus rattus var. Albinus. [En línea]: Revistas. Concytec

(http://revistas.concytec.gob.pe/pdf/scie ntia/v2n1/a02v2n1.pdf

[Consultado 19 mayo 2012].

Cheville N. 1994. Ultrastructural Pathology: The comparative cellular basis of diseases. Iowa State University Press. IA. 946p.

Cornell University. 2009. Plants Poisonous to Livestock: Glucosinolates (Goitrogenic Glycosides).

http://www.ansci.cornell.edu/plants/toxic agents/glucosin.html.

[Consultado junio 01, 2012].

Ferato. 2010. Albúmina.

http://www.ferato.com/wiki/index. php /Alb\%C3\%BAmina

[Consultado 15 septiembre 2011]

Gómez R. 1997. La toxicidad de las plantas ornamentales. 1ra edición. Edit. Oicosfad. Barcelona - España. 199p.

Jínez T, Cortés C, Ávila E, Casaubon T. y Salcedo R. 1998. Efecto de niveles elevados de semilla de jamaica (Hibiscus sabdariffa) en dietas para pollos sobre el comportamiento productivo y funcionamiento hepático.

http://www.medigraphic.com/pdfs/vetme x/vm-1998/vm981f.pdf

[Consultado marzo 30, 2012].

Mateo, R. 2006. El valor hematocrito. http://www.mailxmail.com/curso-analisis linicos-rutina/valor-hematocrito

[Consultado 27 abril 2012]

Miranda S, Rincón $H$, Muñoz $R$, Higuera $A$, Arzálluz A, Urdaneta H. 2007. Parámetros productivos y química sanguínea en pollos de engorde alimentados con tres niveles dietéticos de harina de granos de fríjol (Vigna unguiculata (L. Walp.) durante la fase de crecimiento.

http://redalyc.uaemex.mx/pdf/959/9591 7208.pdf.

[Consultado 15 mayo 2011]

Quintana R. 2009. Inhibición de factores antinutricionales (taninos), presentes en la semilla y torta de sacha inchi (Plukenetia volubilis L) mediante diferentes tratamientos térmicos. Tesis de Ing. Zootecnista. Universidad Nacional Agraria de la Selva, Tingo María. 63p.

Mondragón G. 2009. Estudio farmacognóstico y bromatológico de los residuos industriales de la extracción del aceite de Plukenetia volubilis L. (Sacha inchi). Tesis de Químico Farmacéutica. Universidad Nacional Mayor de San Marcos, Lima. 127p.

Perozo J, Ferrer M, Alvarado H, Mavarez Y. y Gil M. 2003. Valores hematológicos en pollos de engorde expuestos de forma continua a bajas dosis de Aflatoxina b1 En el estado Zulia, Venezuela. Facultad de Ciencias Veterinarias de la Universidad del Zulia.

Quispe M. 2008. Niveles de inclusión de sacha inchi (Plukenetia volubilis L) tostado, en la dieta, sobre el desempeño productivo de pollos de carne. Tesis de Ing. Zootecnista. Universidad Nacional Agraria de la Selva, Tingo María. 47p.

Reátegui V. et al. 2010. Evaluación de la Torta de Sacha Inchi (Plukenetia volubilis) y su uso como fuente alternativa y proteica en la alimentación de pollos de engorde y gallinas de postura en Zungarococha - UNAP. 20p. 
Torres E. 2010. Sustitución parcial de la torta de soya con torta de sacha inchi (Plukenetia volubilis L) precocida, en la dieta sobre el desempeño de pollos de carne (comunicación personal).

Universidad de Zaragoza. 2011. Valores hematológicos normales. [En línea]:Cea.unizar.es(http://cea.unizar.es/ Disenos_experimentales/Sangre/alores\% 20hematologicos.pdf.

[Consultado 15 mayo 2011] 